\title{
N-Alkylacrylamide copolymers with (meth)acrylamide derivatives of cholic acid: Solution properties and aggregation
}

\author{
Damien Avoce, Wilms E. Baille, X. X. Zhu *
}

Département de Chimie, Université de Montréal, C.P. 6128, Succursale Centre-ville, Montréal, Québec, H3C 3J7, Canada; julian.zhu@umontreal.ca

(Received: November 26, 2003; published: January 31, 2004)

\begin{abstract}
Solution properties of a series of thermosensitive copolymers based on acrylamide and methacrylamide derivatives of cholic acid and $\mathrm{N}$-alkylacrylamides such as $\mathrm{N}, \mathrm{N}$-diethylacrylamide, $\mathrm{N}$-ethylacrylamide, and $\mathrm{N}$-isopropylacrylamide have been studied by various techniques including fluorescence and turbidimetry. These copolymers contained $1-5 \mathrm{~mol}-\%$ of the (meth)acrylamide derivatives of cholic acid as determined by NMR spectroscopy. A two-stage aggregation process was observed for the copolymers of $N$-isopropylacrylamide when the number of cholic acid residues was sufficiently high. The presence of the copolymers lowered the critical micellar concentration of sodium dodecyl sulfate but had little effect on the micellization of sodium cholate. The critical micellar concentration of these copolymers decreased with increasing content of cholic acid residues in the polymers.
\end{abstract}

\section{Introduction}

Biocompatibility and toxicity are major concerns for polymers used in biomedicine [1], which can be addressed when natural compounds such as bile acids are incorporated into the polymers [2-5]. Interesting properties can be introduced into the polymers by the inclusion of natural compounds. For example, bile acid containing polymers or polymers bearing alkyl cholesteryl groups can form micelles and their hydrophobic interior can act as a microcontainer for hydrophobic compounds [6,7]. Furthermore, these polymers may be used to remove bile salts from the digestive tract in order to reduce the cholesterol level in blood, thereby lowering the risk of cardio-vascular diseases [8]. The removal of bile salts is possible since bile acid residues attached on the polymer may facilitate the formation of micelles of bile salts thereby hindering the resorption of bile acids and hence leading to the removal of bile salts.

Thermosensitive polymers can be potentially used as additives in pharmaceutical industries [9-11]. Some $N$-isopropylacrylamide (NIPAM) hydrogels can be used as thermally responsive attenuators for ultrasonic waves [12]. NIPAM and methacrylic acid gels can be used for episodic drug delivery [13]. These polymers can undergo a thermally reversible phase separation at a lower critical solution temperature (LCST), known as cloud point (CP) for complex systems. The phase separation is caused by the disruption of hydrogen bonds with water and the hydrophobic aggregation of the 
polymers [14]. Recently, we have reported the synthesis of thermosensitive copolymers with bile acid derivatives [15]. The dependence of the LCST on the structure of $\mathrm{N}$-alkylacrylamide copolymers with bile acid residues has been studied [16]. The inclusion of even small amounts of bile acid derivatives lowers significantly the LCST of the polymers $[15,17]$. To raise the LCST of such polymers, the bile acid derivatives can be copolymerized with more hydrophilic $N$-alkylacrylamides such as $N$-ethylacrylamide (EA), the homopolymer of which exhibited a higher LCST in water $\left(82^{\circ} \mathrm{C}\right)$ $[18,19]$. The aim of the present work is to study the aggregation and solution properties of $\mathrm{N}$-alkylacrylamide copolymers containing bile acid derivatives. We have also studied the effects of surfactants on the aggregation of the copolymers and the effects of copolymers on the micellization of selected surfactants.

\section{Experimental part}

\section{Materials and synthesis}

Cetylpyridinium salt was purchased from Sigma. All other chemicals were purchased from Aldrich and used as received. The (meth)acrylamide derivatives of cholic acid and their copolymers with $\mathrm{N}$-alkylacrylamides were prepared as described in previous reports $[15,16]$.

Fig. 1 shows the chemical structure of copolymers. The acrylamide $(R=H)$ and methacrylamide $\left(\mathrm{R}=\mathrm{CH}_{3}\right)$ derivatives of cholic acid are denoted as ACA and MACA, respectively. $N, N$-Diethylacrylamide is denoted as DEA. The copolymers with these monomers are denoted as poly(NIPAM-ACA), poly(EA-DEA-ACA), poly(NIPAMMACA) or poly(EA-DEA-MACA). The percentage used in the abbreviation of copolymers indicates the mole percent of monomers containing the cholic acid residue. In the terpolymers, the mole fractions of EA and DEA were equal. The chemical compositions of the copolymers were determined by NMR spectroscopy and are listed in Tab. 1.

Size exclusion chromatography (SEC) was used to determine the molecular weight of the copolymers as describes previously [16]. The results obtained indicate a weightaverage molecular weight $\left(M_{\mathrm{w}}\right)$ of $c .58000$ and $M_{\mathrm{w}} / M_{\mathrm{n}}$ of 2.01 for poly(EA-DEAACA $x \%$ ) and poly(EA-DEA-MACA $\%$ ) where $x=0,1,3$. Poly(EA-DEA-ACA5\%) and poly(EA-DEA-MACA5\%) have an $M_{\mathrm{w}}$ of 18000 and an $M_{\mathrm{w}} / M_{\mathrm{n}}$ of 2.01 [16]. As reported previously [15], $M_{\mathrm{w}}$ for NIPAM copolymers was $c$. 100000 and the polydispersity 2.6 - 2.9. Detailed results are shown in Tab. 1.

\section{Cloud point (CP) measurements}

CP measurements were performed on a CARY 1 BIO UV-visible spectrophotometer coupled with a temperature controller. A 1-cm sample cell containing approximately $3 \mathrm{ml}$ of polymer solutions was used against distilled water as reference. The polymer concentrations ranged from 0.1 to $5 \mathrm{wt} . \%$. The solutions were heated from 10 to $50^{\circ} \mathrm{C}$ at $0.5^{\circ} \mathrm{C} / \mathrm{min}$. The $\mathrm{CP}$ is defined as the temperature at which the derivative of absorbance with respect to temperature at a certain wavelength is maximal. To study the effect of added salt, polymer solutions (1 wt.-\%) containing $0-2 \mathrm{M} \mathrm{NaCl}$ in distilled water were prepared. The same $\mathrm{NaCl}$ solutions without the polymer were used as a reference. The wavelength was fixed at $500 \mathrm{~nm}$. Sodium dodecyl sulfate (SDS) and sodium cholate ( $\mathrm{NaC}$ ) were used as surfactants. The concentration of the surfactants varied from 0 to $2.5 \mathrm{mM}$ for SDS and from 0 to $20 \mathrm{mM}$ for $\mathrm{NaC}$. 


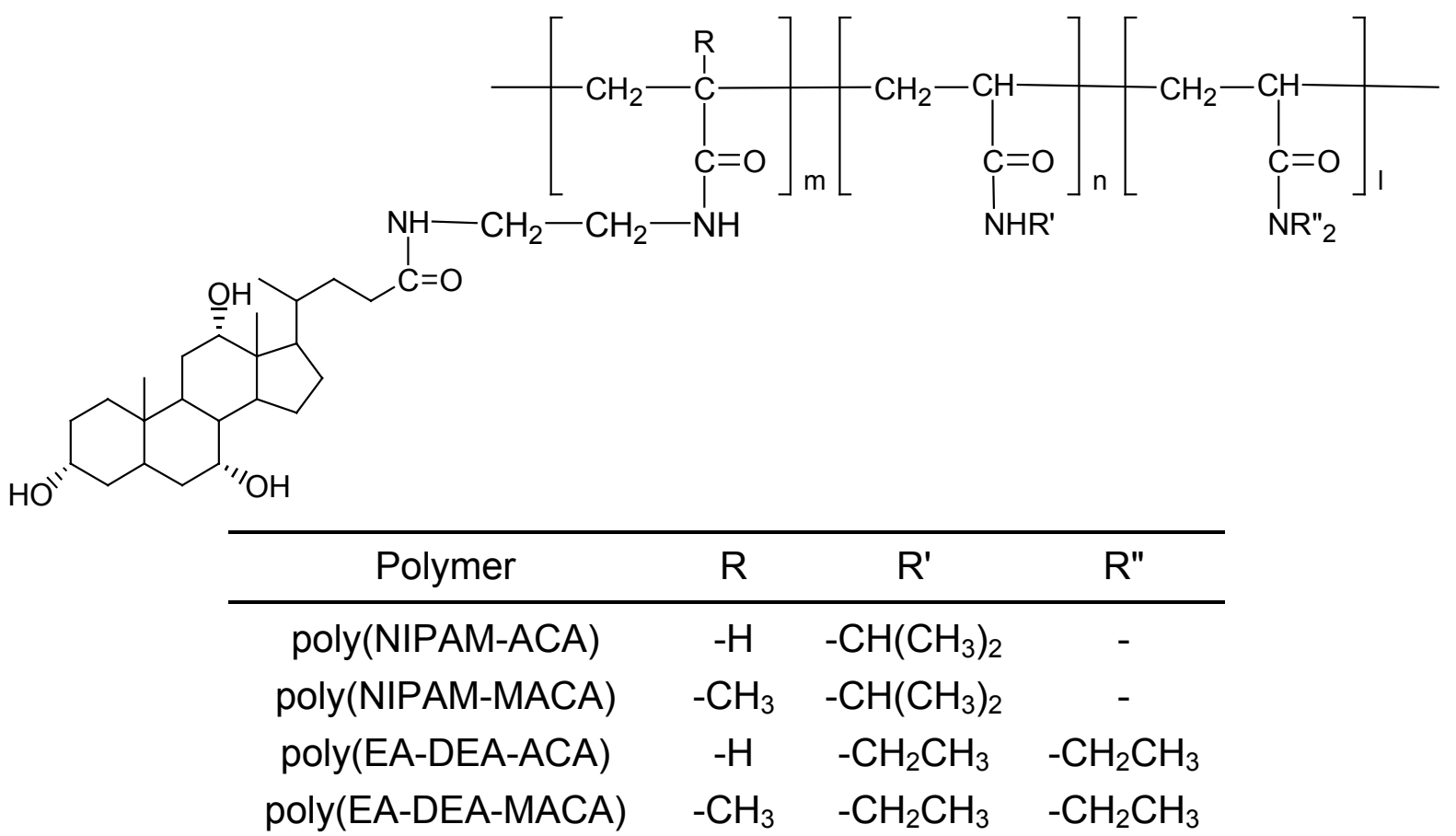

Fig. 1. The chemical structure of $\mathrm{N}$-isopropylacrylamide copolymers and $\mathrm{N}$-ethylacrylamide and $\mathrm{N}, \mathrm{N}$-diethylacrylamide copolymers with (meth)acrylamide derivatives of bile acids

Tab. 1. The chemical composition, molecular weight and CMC of the copolymers containing (meth)acrylamide derivatives of cholic acid

\begin{tabular}{cccc}
\hline Polymer & $\begin{array}{c}\text { Composition } \\
\text { (mole ratio) }\end{array}$ & $M_{\mathrm{n}}$ & CMC in wt.-\% \\
\hline Poly(EA-DEA) & $50.0: 50.0$ & 58000 & 0.30 \\
Poly(EA-DEA-ACA1\%) & $49.5: 49.5: 1$ & 58000 & 0.0038 \\
Poly(EA-DEA-ACA3\%) & $48.5: 48.5: 2.9$ & 58000 & 0.0038 \\
Poly(EA-DEA-ACA5\%) & $47.6: 47.6: 4.8$ & 18000 & 0.0022 \\
PNIPAM & - & 100000 & - \\
Poly(NIPAM-ACA1\%) & $99.0: 1.0$ & 86400 & - \\
Poly(NIPAM-ACA3\%) & $97.1: 2.9$ & 109200 & - \\
Poly(NIPAM-ACA5\%) & $95.3: 4.7$ & 99300 & -
\end{tabular}

\section{Fluorescence measurements}

To determine the critical micellar concentrations (CMC) of the polymers in water and their effect on the micellization of surfactants, fluorescence measurements of pyrene probes were carried out [20]. Steady-state fluorescence spectra were recorded at room temperature on a Perkin-Elmer LS 50B spectrofluorimeter using narrow $(0.2$ $\mathrm{mm}$ ) slits. An excitation wavelength at $336 \mathrm{~nm}$ was used. The spectra were recorded from 360 to $700 \mathrm{~nm}$ at $90 \mathrm{~nm} / \mathrm{min}$. Stock solutions of $20 \mathrm{mM}$ bile salt in a $1.7 \mu \mathrm{M}$ pyrene solution in Millipore water were first prepared. A stock solution of polymer in 1.7 $\mu \mathrm{M}$ aqueous pyrene solution was then prepared. Samples were prepared by 
dilution of the bile salt stock solution with the $1.7 \mu \mathrm{M}$ aqueous pyrene solution to the desired concentrations. Solutions of the bile salt and the polymer were prepared by mixing the appropriate stock solutions and then diluting them to the desired concentrations. Solutions were not degassed prior to use [21]. The $\mathrm{pH}$ of prepared salt solutions remained constant at about 8 after addition of the polymer and the fluorescent probe.

\section{Results and discussion}

Some examples of NMR spectra of the copolymers have been published and commented in previous reports $[15,16]$.

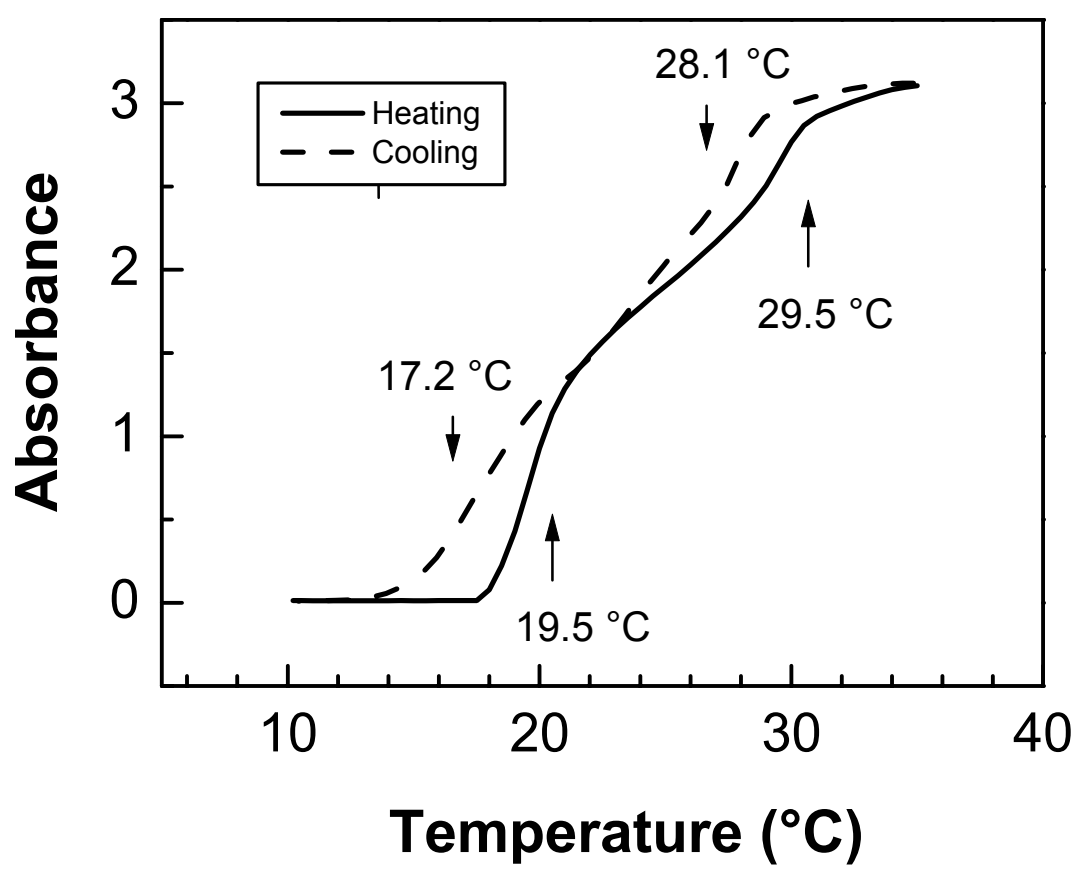

Fig. 2. The reversibility of the two-stage aggregation of poly(NIPAM-MACA3\%) (1 wt.- $\%$ in water, heating and cooling rate are both $0.5^{\circ} \mathrm{C} / \mathrm{min}$ ) is clearly shown. The discrepancy of the temperatures at which the aggregations occurred during cooling and heating is also shown

\section{$\mathrm{CP}$ of $\mathrm{N}$-alkylacrylamide copolymers}

We have reported that the CP of the (meth)acrylamide copolymers depends on the chemical composition and that the CP decreases with increasing content of bile acid residues in the copolymers as for other $\mathrm{N}$-substituted alkylacrylamide copolymers $[15,16]$. Furthermore, in the case of the copolymers of NIPAM with (meth)acrylamide derivatives of cholic acid, we observed a two-stage aggregation process when the temperature was raised [15]. Fig. 2 shows this aggregation process obtained for poly(NIPAM-MACA $3 \%$ ). The first step of the aggregation at a lower temperature is shown by a sharp increase in turbidity of the solution, followed by a more gradual increase in the turbidity, which corresponds more closely to the CP value measured by calorimetry [16]. Fig. 2 also shows that the two-stage aggregation process is reversible. This reverse process is illustrated by the dashed line. Furthermore, the two steps in the reversible process illustrate a more gradual decrease in the turbidity. The temperatures at which the two-stage aggregations occurred during the cooling 
process are lower than those during heating. This is probably due to the kinetic difference between formation and dissolution of the aggregates during the heating and cooling processes. The first step may be due to the aggregation of the bile acid derivative and the second may be due to the further aggregation of the hydrophobic part of alkylacrylamide monomer units. However, such a two-stage process was not observed for the copolymers with EA.

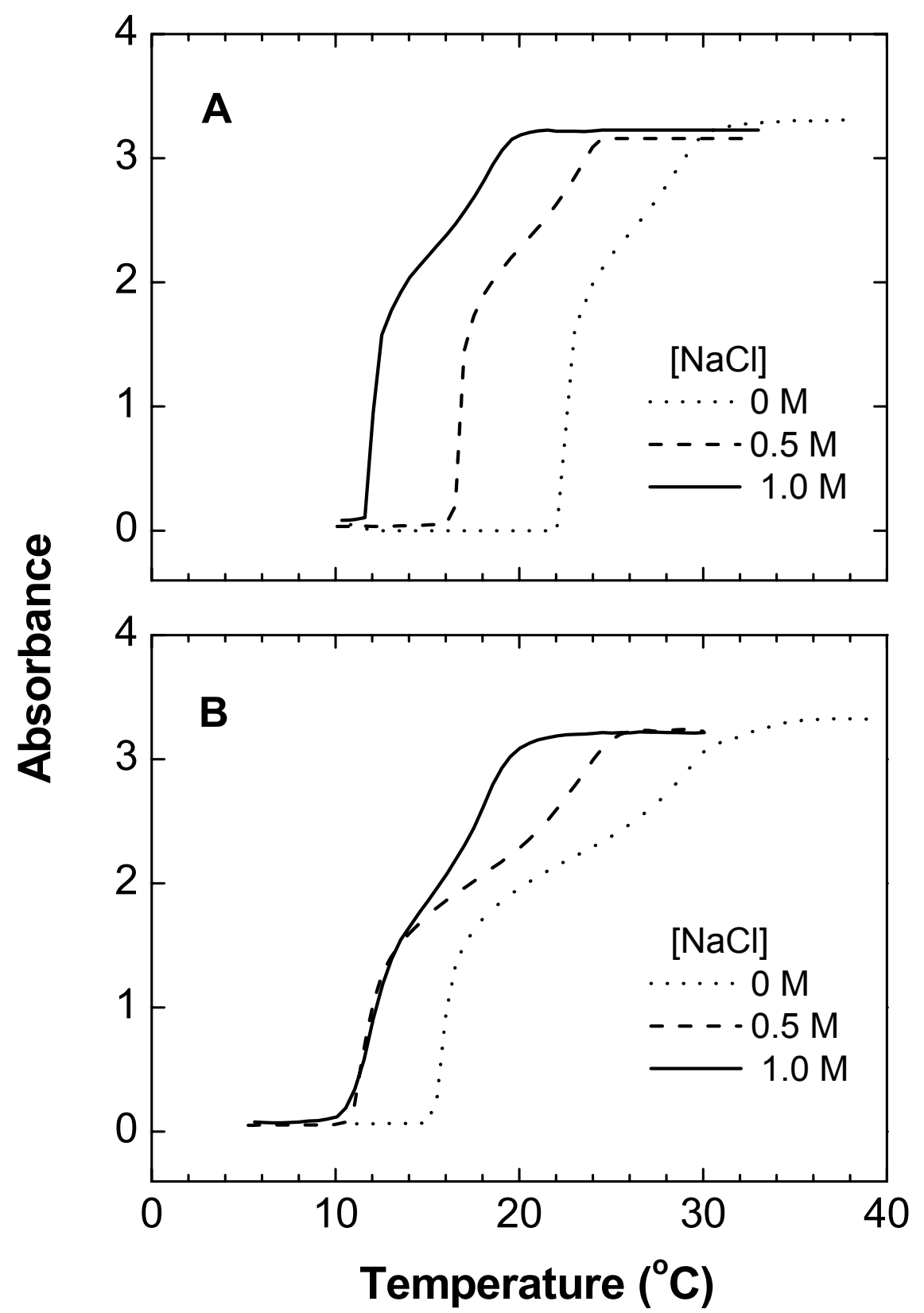

Fig. 3. The effect of added $\mathrm{NaCl}$ on the cloud point of (A) poly(NIPAM-ACA3\%), and (B) poly(NIPAM-MACA3\%)

\section{Effect of added salts}

In the design of thermoselective sustained release devices, the permeability of the polymer under different conditions is important [10]. It is thus interesting to study the effect of added salts on the properties of the polymer solutions. The inorganic salt ions can disrupt hydrogen bonds, break up the ordered water structure and promote 
hydrophobic polymer-polymer interactions. As expected, Fig. 3 shows that the addition of $\mathrm{NaCl}$ lowered the CPs of poly(NIPAM-ACA3\%) and poly(NIPAMMACA3\%), but more significantly than in the case of PNIPAM as reported in the literature [22-24]. The salt effect is even more pronounced for poly(NIPAM-ACA) than for poly(NIPAM-MACA) (Fig. 3). Even with added salt, the two stages in the aggregation process are still evident, but they occur at lower temperatures. However, at high concentrations of added salt in the system, the two steps tend to merge into one. This is quite evident for poly(NIPAM-MACA) (Fig. 3B). This trend may be due to the fact that when the salt concentration is high enough, the difference in hydrophobicity between the $\mathrm{N}$-alkyl group and the bile acid residues in the copolymers is not significant any more.

For the series of EA-DEA copolymers, we selected poly(EA-DEA) and poly(EA-DEAACA $1 \%$ ) to study the effect of sodium chloride. Fig. 4 illustrates the variation of CP with the addition of salt into the polymer solutions. For the two cases, CP decreases almost exponentially with increasing amount of the salt. In comparison, previous results obtained for DEA homopolymer showed a linear dependence of the CP on the $\mathrm{NaCl}$ concentration [22]. The difference between the homo- and copolymers is not understood. This is probably related to the added hydrophilicity of EA in the copolymers. Obviously, the presence of even a small amount of cholic acid residues (1 mol-\%) lowers the CP of the copolymers.

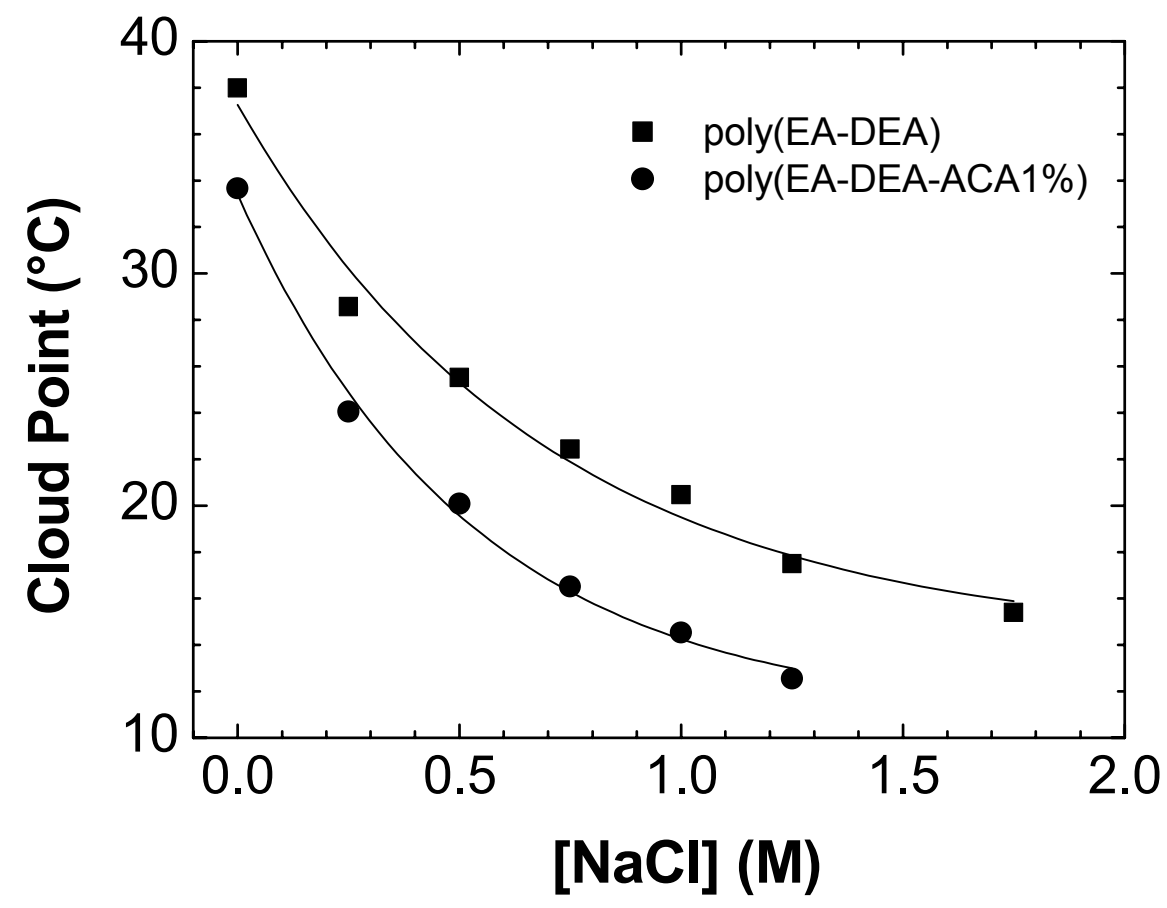

Fig. 4. The effect of added $\mathrm{NaCl}$ on the cloud point of copolymers as studied by UVvisible absorbance. [polymer] $=1 \mathrm{wt} . \%, V_{H}=0.5^{\circ} \mathrm{C} / \mathrm{min}$ and $\lambda=500 \mathrm{~nm}$. Poly(EADEA): squares; poly(EA-DEA-ACA1\%): circles

\section{Effect of added surfactants}

UV-visible spectrophotometry was used to study the polymer solutions upon the addition of appropriate amounts of a surfactant $(0-7 \mathrm{mM})$. The reference cell was filled with the water solution of SDS. The CP of the NIPAM copolymers with ACA and MACA increases with the addition of SDS into the solution (data not shown). The 
two-stage aggregation merged into one step when SDS was added in relatively larger amounts (above $1 \mathrm{mM}$ ). At higher concentrations of SDS, the solution tended to remain clear because of the emulsification of the polymer by the surfactant and a CP was no longer observed. However, the concentration of SDS was still far below its CMC (c. $8.3 \mathrm{mM}[25])$.

Fig. 5 shows the effect of SDS on the CP of EA-DEA copolymers. The CP for all the polymers increased continuously with increasing concentration of SDS and seemed to converge into one single value at about $49^{\circ} \mathrm{C}$, after which the $\mathrm{CP}$ was no longer detectable because the solutions tended to remain clear. The results also show that SDS raised more significantly the CP of the copolymers with higher content of bile acid residues. The UV-visible spectra of polymer solutions show that when $\lambda$ is above $450 \mathrm{~nm}$, the absorbance of the solution with SDS is significantly lower than the absorbance of the solution without surfactant. Obviously, the hydrophobic interactions with the surfactant are more effective for the bile acid moiety in the polymers and the solubilization effect of the soap lowers the CP of the polymers and improves the clarity of their solutions. In the fluorescence study of water-soluble polymers such as poly ( $N$-vinylpyrrolidone) and poly(ethylene oxide) by Turro et al. [26], two stages of the interaction were observed. The first step is an aggregation of the surfactant along the polymer chain and the second is a process of complete micellization of surfactant $[26,27]$. The behaviour of the systems studied here probably corresponds to the first step only since the concentration of the surfactant is not yet sufficiently high.

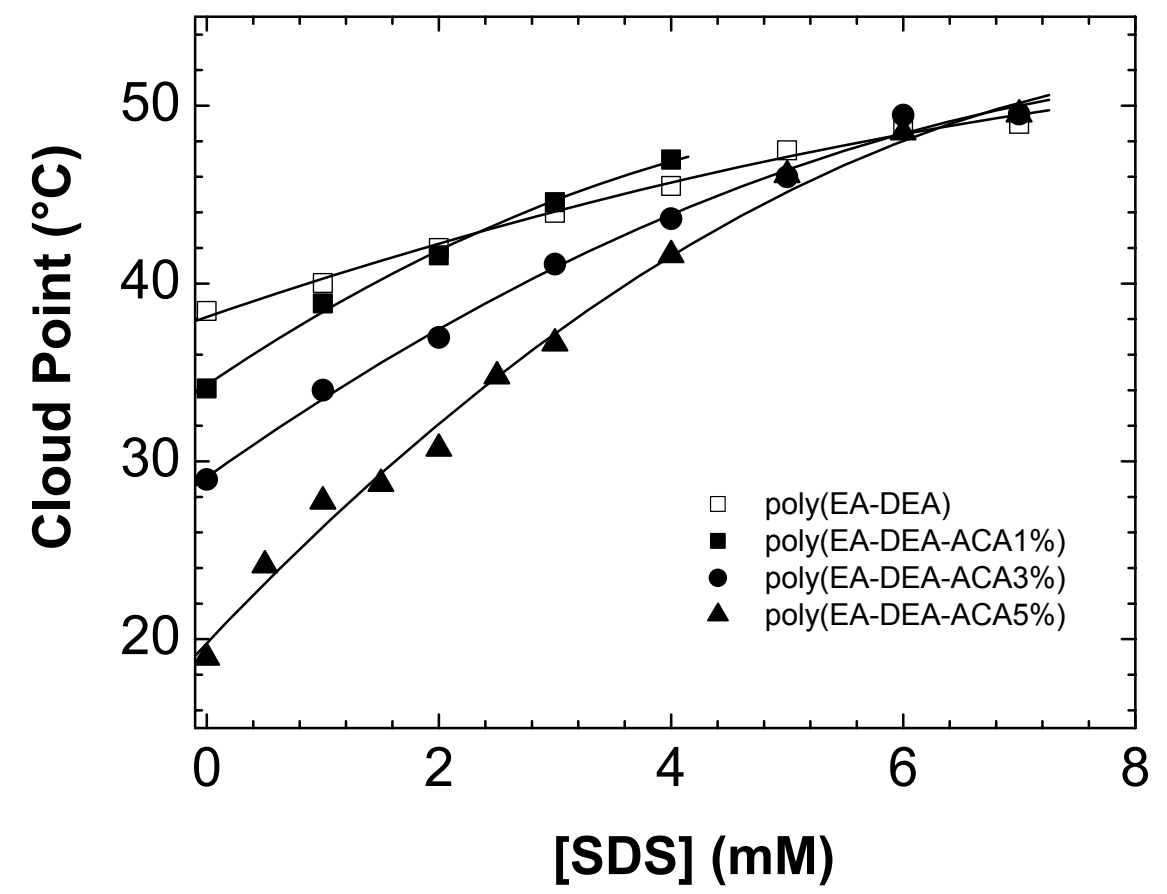

Fig. 5. The effect of SDS on the cloud point of poly(EA-DEA) and poly(EA-DEAACA $x \%$ ). Aqueous solutions were studied by UV-spectroscopy. [polymer] $=1 \mathrm{wt} . \%$, $V_{\mathrm{H}}=0.5^{\circ} \mathrm{C} / \mathrm{min}$ and $\lambda=500 \mathrm{~nm}$

The formation of bile salt micelles is important for the enterohepatic circulation of bile salts and the digestion of lipids [8,28-30]. The effect of bile salts on the CP of the polymer solutions was studied by UV-visible spectrophotometry. The polymer concentration was set at $1 \mathrm{wt} .-\%$ and the heating rate was fixed at $0.5^{\circ} \mathrm{C} / \mathrm{min}$. The 
size of the aggregates did not seem to vary as shown by the absorbance spectra of poly(NIPAM-MACA3\%) solution with added sodium cholate (NaC) recorded at different temperatures. Only a one-step aggregation was detected. A linear dependence is observed for copolymers with $x$ less than or equal to 3 , where the CP increased slightly with increasing $\mathrm{NaC}$ concentration (data not shown). More than 20 $\mathrm{mM}$ of $\mathrm{NaC}$ need to be added to increase the $\mathrm{CP}$ for about $2-5^{\circ} \mathrm{C}$. The most significant effect is on poly(EA-DEA-ACA5\%); its CP increased c. $10^{\circ} \mathrm{C}$ when $7 \mathrm{mM} \mathrm{NaC}$ was added. The solubilization effect of $\mathrm{NaC}$ is lower than of a good surfactant such as SDS and its effect on the CP of the thermosensitive polymers is correspondingly weaker.

\section{The critical micellar concentration (CMC) of the polymers}

The CMC of the polymers has been studied. As reported previously [15,17], the CMC of such copolymers decreases with increasing content in bile acid residue. We have shown previously [17] that the CMC of the copolymers of NIPAM and bile acid derivatives is very low (about 0.0012 wt.-\%) for a low content of bile acid residues in the copolymers. The copolymers we studied here are more hydrophilic and have higher $\mathrm{CP}$. It is interesting to study their aggregation properties by fluorescence. We chose a temperature at which all our polymers remained soluble $\left(15^{\circ} \mathrm{C}\right)$.

Fluorescent dyes such as pyrene can be used to study the interaction between the surfactants and the polymers. Fig. 6A shows an example of a fluorescence spectrum of pyrene in a solution of poly(NIPAM-MACA3\%) with $32 \mathrm{mM}$ of $\mathrm{NaC}$. The steadystate fluorescence spectrum of pyrene has five different vibronic bands. The first near $372 \mathrm{~nm}$ shows enhanced fluorescence intensities in a more polar environment while the third vibronic band near $384 \mathrm{~nm}$ remains insensitive to the change of environment $[20,31]$. The ratio of the maximum peak intensity $I_{3} / I_{1}$ increases with the polarity of the environment. An abrupt change of $\mathrm{I}_{3} / \mathrm{l}_{1}$ indicates the formation of micelles. Goddard [31] and Winnik et al. [21] believed that the CMC is situated between the concentration at which the interaction between the surfactant and the polymer first occurs and the concentration at which the polymer becomes saturated with surfactants.

To determine the CMC of the polymers we prepared different solutions of copolymers from 0.0004 to $1 \mathrm{wt} .-\%$. The excitation wavelength was set at $336 \mathrm{~nm}$. The CMC was defined as the polymer concentration at which the derivative of $I_{3} / l_{1}$ with respect to the polymer concentration was maximal. For both series of NIPAM copolymers (ACA and MACA), the micellization at $15^{\circ} \mathrm{C}$ starts at very low concentrations (about 0.0014 wt.-\%), while the micellization of PNIPAM was not observed up to a concentration of 0.04 wt.-\% [25]. The CMC of the copolymers is obviously lowered by the presence of the bile acid residues in the polymer.

In Fig. 6B, one can see that the CMC of the EA-DEA copolymers decreases also with increasing content of bile acid residues in the copolymers. For example, the CMC of poly(EA-DEA) is 0.30 wt.- $\%$ and for poly(EA-DEA-ACA5\%) it is 0.0022 wt.- $\%$. Kim et al. reported a CMC of $0.01 \mathrm{wt} . \%$ for some copolymers with bile acid pendants and NIPAM [32]. The values of CMC in this study are much higher than the values reported in the literature for similar but less hydrophilic copolymers [17]. 

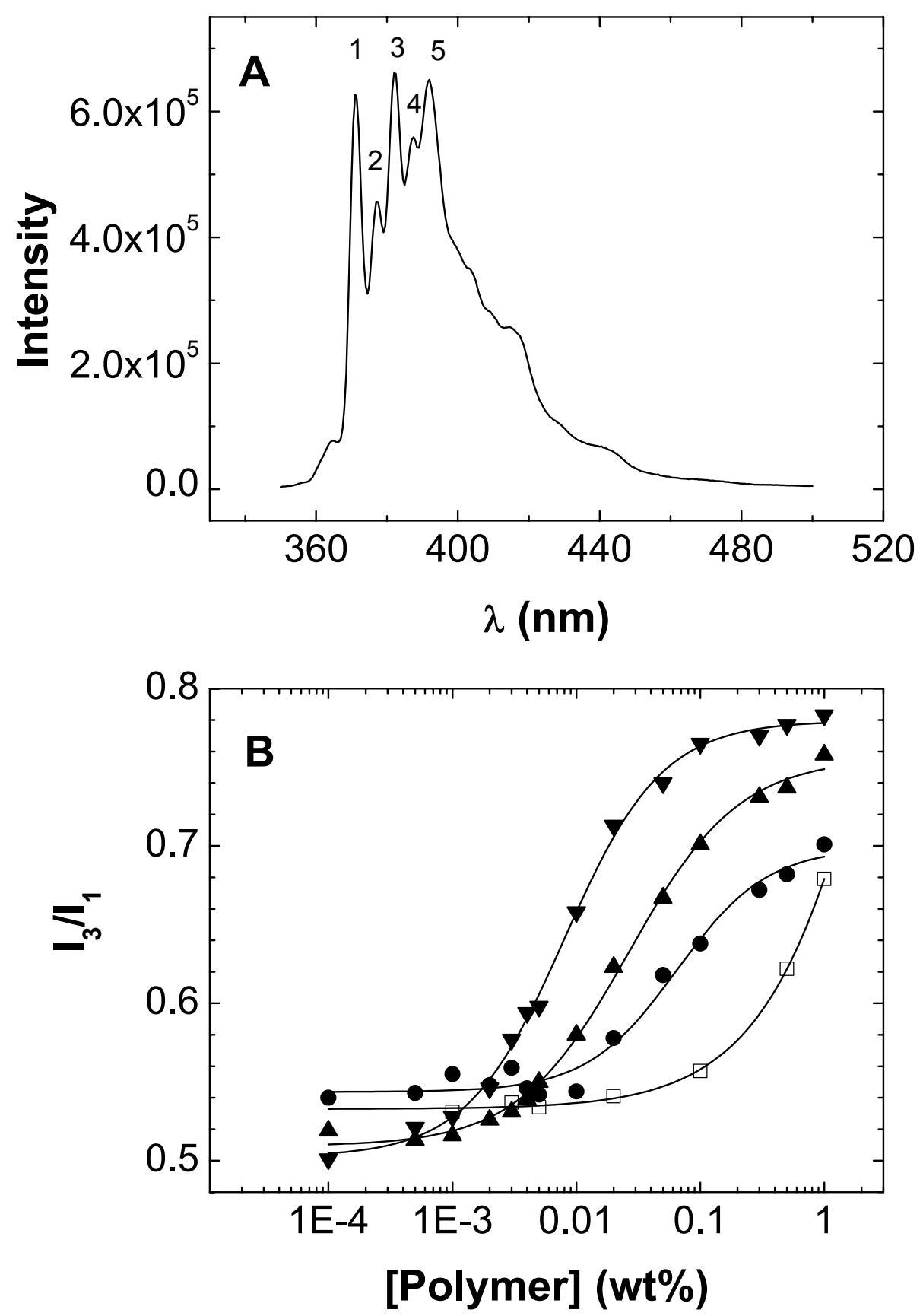

Fig. 6. (A) The fluorescence spectrum of pyrene in a poly(NIPAM-ACA3\%) aqueous solution. $[$ pyrene $]=1.7 \mu \mathrm{M},[\mathrm{NaC}]=32 \mathrm{mM}$, [poly $($ NIPAM-ACA3\%) $]=0.0012 \mathrm{wt} .-\%$. (B) The effect of the copolymer composition on the CMC of poly(EA-DEA-ACAx\%), where $x=0$ : squares, $x=1$ : circles, $x=3$ : upward triangles, and $x=5$ : downward triangles. $\lambda_{\mathrm{ex}}=336 \mathrm{~nm} ; T=15^{\circ} \mathrm{C}$, [pyrene] $=1.7 \mu \mathrm{M}$

\section{Effect of the copolymers on the micellization of surfactants}

Poly(EA-DEA-ACA3\%) was selectively used to study the effect of copolymers on the micellization of surfactants. Since the copolymers can also self-aggregate, their effect on the micellization of surfactants cannot be studied above their CMC (c. 0.0038 wt.-\%). Therefore, three concentrations of copolymer $(0.001,0.002$, and 0.003 wt.- $\%$ ) were used. Fluorescence studies were carried out at $15^{\circ} \mathrm{C}$ (below the $\mathrm{CP}$ of the more hydrophobic copolymer) to avoid the precipitation of the copolymer. Fig. 7 shows the $I_{3} / l_{1}$ dependence on the SDS concentration. 


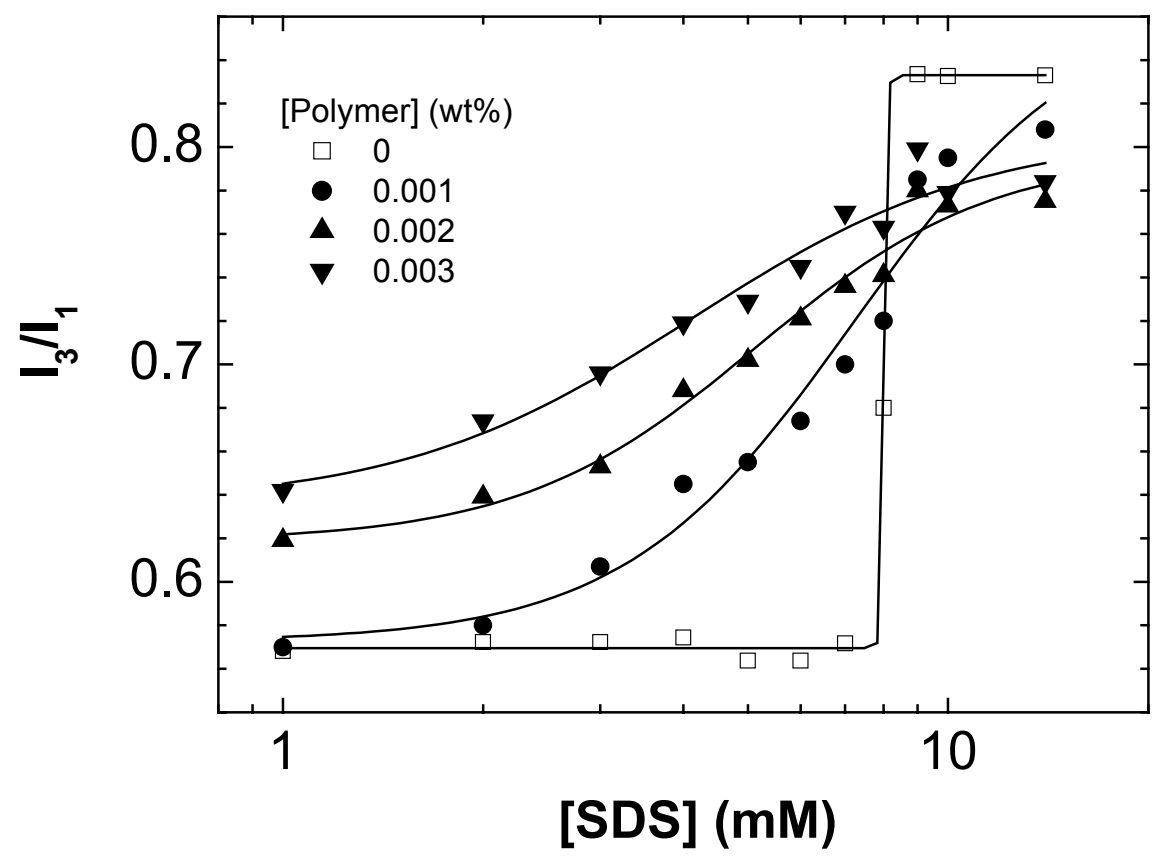

Fig. 7. The effect of the polymer concentration on the micellization of SDS by poly(EA-DEA-ACA3\%). [pyrene] $=1.7 \mu \mathrm{M}, \lambda_{\mathrm{ex}}=336 \mathrm{~nm}, T=15^{\circ} \mathrm{C}$

\section{The effect on SDS}

The critical aggregation concentration (CAC) of a surfactant is defined as the concentration at which it starts to react with the polymer. The CAC of the surfactants can be taken from the intersection of the tangent to the curve at the inflection with the horizontal tangent through the points of low concentration [32]. Before the addition of polymers, the CMC of SDS is reflected by a sharp change of $I_{3} / l_{1}$ at about $8.2 \mathrm{mM}$. This is comparable to the results of Schild and Tirrell [25] (8.3 mM), Biggs et al. [33] and Majhi et al. [34] (8.2 - 8.6 mM). As shown in Fig. 7, with the addition of poly(EADEA-ACA3\%) to the solution, the CAC tends to decrease with increasing polymer concentration. Poly(NIPAM-ACA3\%) has a very similar effect (data not shown). The aggregation of the surfactant on the polymer starts before the CMC of the surfactant. Previous studies indicated that the surfactant molecules (SDS) bind to the polymer (PNIPAM) and the process is cooperative $[21,25]$. Several observations can be made in Fig. 7. First, it is clear that the presence of the polymer lowers the micellization concentration of SDS. The lowering of the micellization concentration depends on the amount of added polymer. Second, the micellization of the surfactant occurs over a wider range of concentrations with the addition of the polymer (the shapes of the curves become less abrupt than those of the surfactant alone). Third, the CAC of the surfactant is difficult to determine from these curves. These results are similar to those obtained by Biggs et al. [33] for copolymers of acrylamide and $\mathrm{N}$-(4-ethylphenyl)acrylamide, which lowered the CMC of SDS.

The effect on sodium cholate $(\mathrm{NaC})$

The study of the micellization of bile salts can help to gain a better understanding of their interactions with biological membranes and cholesterol. Therefore, we also studied the effect of the copolymers on the micellization of $\mathrm{NaC}$. The concentration of bile salts in the gallbladder is about $10-50 \mathrm{mM}$ and outside the gallbladder this concentration is less than $4 \mathrm{mM}$ [32]. We selected the concentration of bile salt at 
lower than $20 \mathrm{mM}$. The $\mathrm{CMC}$ of $\mathrm{NaC}$ was measured to be about $13.5 \mathrm{mM}$, a value quite close to those in the literature $(13.5-13.9 \mathrm{mM})[20,34]$. It is known that the addition of bile acid dimers lowered the CMC of $\mathrm{NaC}$ [20]. Similar copolymers did not show any effect on the micellization of $\mathrm{NaC}$ [17]. The addition of these copolymers in this concentration range did not induce any change of the $\mathrm{CMC}$ of $\mathrm{NaC}$, nor of the shape of the curves. However, the ratios $I_{3} / l_{1}$ for $\mathrm{NaC}$ without or with the copolymers are higher $(0.6-1.2)$ than those of classical micelles such as SDS (0.5 - 0.8) [35].

\section{Conclusions}

(Meth)acrylamides containing cholic acid residues can be introduced into the polymers and copolymers of $\mathrm{N}$-substituted acrylamides. The CPs of the copolymers are lowered as a consequence. When the content of cholic acid residues is sufficiently high, a reversible two-stage aggregation behaviour was observed due to the aggregations of the cholic acid derivatives prior to the hydrophobic aggregation of the $\mathrm{N}$-substituted alkylacrylamide monomer units. The two-stage aggregation process may be potentially useful for the application of such polymers such as controlled release devices. The addition of $\mathrm{NaCl}$ decreased the $\mathrm{CP}$ of the aqueous solutions of the copolymers as expected, but always with the presence of a two-stage aggregation. The addition of SDS increased the CP and merged the two aggregations into one. The presence of the copolymers lowered the CMC of SDS (and broadened the micellization to a larger concentration range) but had little effect on the micellization of cholic acid in the aqueous environment. It may be interesting to study the binding of bile salts by the copolymers above their CMC when the polymers are already selfaggregated.

Acknowledgement: Financial support from the Natural Sciences and Engineering Research Council (NSERC) of Canada and Fonds FCAR of the Province of Quebec is gratefully acknowledged.

[1] Clas, S.-D.; Yapp, D. T. T.; Zhu, X. X.; Recent Res. Devel. Macromol. Res. 1998, 3, 475.

[2] Ahlheim, M.; Hallensleben, M. L.; Makromol. Chem., Rapid Commun. 1988, 9, 299.

[3] Denike, J. K.; Zhu, X. X.; Macromol. Rapid Commun. 1994, 15, 459.

[4] Zhu, X. X.; Moskova, M.; Denike, J. K.; Polymer 1996, 27, 493.

[5] Zhang, Y. H.; Akram, M.; Liu, H. Y.; Zhu, X. X.; Macromol. Chem. Phys. 1998, 99, 1399.

[6] Kim, I.-S.; Jeong, Y.-I.; Kim, S.-H.; Arch. Pharm. Res. 2000, 23, 87.

[7] Tsuchida, E.; Yamamoto, K.; Miyatake, K.; Endo, K.; Macromolecules 1997, 30, 4235.

[8] Ahlheim, M.; Hallensleben, M. L.; Makromol. Chem. 1992, 193, 779.

[9] Yamazaki, A.; Song, J.; Winnik, F. M.; Brash, J. L.; Macromolecules 1998, 31, 109.

[10] Okahata, Y.; Noguchi, H.; Seki, T.; Macromolecules 1986, 19, 494. 
[11] Chiu, H.-C.; Chern, C.-S.; Lee, C.-K.; Chang, H.-F.; Polymer 1998, 39, 1609.

[12] Yuan, K.; Hu, Z.; Li, Y.; Appl. Phys. Lett. 1999, 74, 2233.

[13] Siegel, R. A.; Misra, G. P.; Polym. Prepr. (Am. Chem. Soc., Div. Polym. Chem.) 2000, 41, 705.

[14] Boutris, C.; Chatzi, E. G.; Kiparissides, C.; Polymer 1997, 38, 2567.

[15] Liu, H.; Avoce, D.; Song, Z.; Zhu, X. X.; Macromol. Rapid Commun. 2001, 22, 675.

[16] Avoce, D.; Liu, H. Y.; Zhu, X. X.; Polymer 2003, 44, 1081.

[17] Benrebouh, A.; Avoce, D.; Zhu, X. X.; Polymer 2001, 42, 4031.

[18] Taylor, L. D.; Cerankowski, L. D.; J. Polym. Sci., Polym. Chem. Ed. 1975, 13, 2551.

[19] Plate, N. A.; Lebedeva, T. L.; Valuev, L. I.; Polym. J. 1999, 31, 21.

[20] Gouin, S.; Zhu, X. X.; Langmuir 1998, 14, 4025.

[21] Winnik, F. M.; Ringsdorf, H.; Venzmer, J.; Langmuir 1991, 7, 905.

[22] Idziak, I.; Avoce, D.; Lessard, D.; Gravel, D.; Zhu, X. X.; Macromolecules 1999, 32, 1260.

[23] Fujishige, S.; Kubota, K.; Ando, I.; J. Phys. Chem. 1989, 93, 3311.

[24] Schild, H. G.; Tirrell, D. A.; J. Phys. Chem. 1990, 94, 4352.

[25] Schild, H. G.; Tirrell, D. A.; Langmuir 1991, 7, 665.

[26] Turro, N. J.; Baretz, B. H.; Kuo, P.-L.; Macromolecules 1984, 17, 1321.

[27] Bahadur, P.; Dubin, P.; Rao, Y. K.; Langmuir 1995, 11, 1951.

[28] Mayes, P. A.; "Digestion/Absorption in the Gastrointestinal Tract", in 'Haper's Review of Biochemistry', $19^{\text {th }}$ ed., Martin, D. W.; Mayes, P. A.; Rodwel, V. W.; editors; Lange Medical Publications, Los Altos 1983, p. 546.

[29] Small, D. M.; "The physical chemistry of cholanic acids", in 'The Bile Acids', Nair, P. P.; Kritchevsky, D.; editors; Plenum Press, New York 1971, vol. 1, p. 249.

[30] Garidel, P.; Hildebrand, A.; Neubert, R.; Blume, A.; Langmuir 2000, 16, 5267.

[31] Goddard, E. D.; Colloids Surf. 1986, 19, 255.

[32] Kim, I.-S.; Jeong, Y.-I.; Kim, S.-H.; Arch. Pharm. Res. 2000, 23, 87.

[33] Biggs, S.; Selb, J.; Candau, F.; Langmuir 1992, 8, 838.

[34] Majhi, P. R.; Moulik, S. P.; Langmuir 1998, 14, 3986.

[35] Zana, R.; Guveli, D.; J. Phys. Chem. 1985, 89, 1687. 